\title{
AATR an ionospheric activity indicator specifically based on GNSS measurements
}

\author{
José Miguel Juan $^{1, *}$, Jaume Sanz ${ }^{1}$, Adrià Rovira-Garcia ${ }^{1}$, Guillermo González-Casado ${ }^{1}$, D. Ibáñez ${ }^{1}$ \\ and R. Orus Perez ${ }^{2}$ \\ ${ }^{1}$ Research Group of Astronomy and Geomatics (gAGE) Universitat Politècnica de Catalunya (UPC) Jordi Girona 1-3, \\ 08034 Barcelona, Spain \\ ${ }^{2}$ Wave Interaction and Propagation Section (TEC-EFW) European Space Agency (ESA ESTEC) Keplerlaan 1, \\ 2201AZ Noordwijk, The Netherlands
}

Received 19 June 2017 / Accepted 6 December 2017

\begin{abstract}
This work reviews an ionospheric activity indicator useful for identifying disturbed periods affecting the performance of Global Navigation Satellite System (GNSS). This index is based in the Along Arc TEC Rate (AATR) and can be easily computed from dual-frequency GNSS measurements. The AATR indicator has been assessed over more than one Solar Cycle (2002-2017) involving about 140 receivers distributed world-wide. Results show that it is well correlated with the ionospheric activity and, unlike other global indicators linked to the geomagnetic activity (i.e. $D_{S T}$ or $A p$ ), it is sensitive to the regional behaviour of the ionosphere and identifies specific effects on GNSS users. Moreover, from a devoted analysis of different Satellite Based Augmentation System (SBAS) performances in different ionospheric conditions, it follows that the AATR indicator is a very suitable mean to reveal whether SBAS service availability anomalies are linked to the ionosphere. On this account, the AATR indicator has been selected as the metric to characterise the ionosphere operational conditions in the frame of the European Space Agency activities on the European Geostationary Navigation Overlay System (EGNOS). The AATR index has been adopted as a standard tool by the International Civil Aviation Organization (ICAO) for joint ionospheric studies in SBAS. In this work we explain how the AATR is computed, paying special attention to the cycle-slip detection, which is one of the key issues in the AATR computation, not fully addressed in other indicators such as the Rate Of change of the TEC Index (ROTI). After this explanation we present some of the main conclusions about the ionospheric activity that can extracted from the AATR values during the above mentioned long-term study. These conclusions are: (a) the different spatial correlation related with the MOdified DIP (MODIP) which allows to clearly separate high, mid and low latitude regions, (b) the large spatial correlation in mid latitude regions which allows to define a planetary index, similar to the geomagnetic ones, (c) the seasonal dependency which is related with the longitude and (d) the variation of the AATR value at different time scales (hourly, daily, seasonal, among others) which confirms most of the well-known time dependences of the ionospheric events, and finally, (e) the relationship with the space weather events.
\end{abstract}

Keywords: positioning system / Total Electron Content (TEC) / ionosphere (general) / algorithm / space weather

\section{Introduction}

The Earth ionosphere is defined as the upper part of the atmosphere where ions and free electrons are present in quantities sufficient to affect the propagation of radio waves (IEEE, 1997). The electron densities are affected by Solar phenomena and the Earth space environment, which includes the Solar wind, the magnetosphere and the geomagnetic field (Zolesi \& Cander, 2014). The identification of perturbed

\footnotetext{
*Corresponding author: jose.miguel. juan@upc.edu
}

periods and regions within such a complex medium is vital to space-based applications, such as the Global Navigation Satellite System (GNSS).

The current and well-established space-weather indices used to characterise the ionospheric activity can be classified according to the scale of their extension. On one hand, global indices linked to the Sun (e.g., the Sun Spot Number (SSN) or the F10.7 centimetre Solar radio flux) are well suited to describe the total ionization level of the ionosphere (Hathaway, 2010); or linked to the Earth magnetic field (e.g., $K_{p}, A_{p}, D_{S T}$ ) which is perturbed by changes in the Solar wind (Rostoker, 1972). 
On the other hand, local indices measure the ionospheric conditions along the Line Of Sight (LOS) of a particular satellite-station pair. Indeed, amplitude and phase fluctuations in the GNSS signals are studied with the $S_{4}$ (Briggs \& Parkin, $1963)$ and the $\sigma_{\phi}$ (Yeh \& Chao-Han, 1982) indices, respectively. In addition, the time derivative of the Total Electron Content (TEC) sampled by dual-frequency receivers was shown capable of measuring the effects of ionospheric irregularities on GNSS signals (Doherty et al., 1994). Indeed, the Rate Of change of the TEC Index (ROTI) defined in Pi et al. (1997), has become one of the most widely-used ionospheric indicators to characterise the ionospheric fluctuations.

The focus of the present paper is to assess a regional ionospheric perturbation index. Several indexes have been defined with distinct degree of usability. For instance, indexes based on the comparison of TEC measurements with respect to non-perturbed reference TEC values such as a background TEC model or monthly median (see, for instance Jakowski et al., 2006; Gulyaeva \& Stanislawska, 2008).

The aviation community recognized the importance of characterising ionospheric threats (i.e., spatial and temporal TEC gradients) to high-integrity augmentation systems (Walter et al., 2001). At a regional scale, the impact of the ionospheric activity on Satellite Based Augmentation System (SBAS) performance was related to the aforementioned geomagnetic Disturbance Storm Time $\left(D_{S T}\right)$ index (see Datta-Barua et al., 2005). At a local scale, ionospheric threats for Ground Based Augmentation System (GBAS) were analysed, modelled and mitigated (Pullen et al., 2009).

The Along Arc TEC Rate (AATR) index (Sanz et al., 2014) was developed in the context of ionospheric studies ${ }^{1}$ for the European Geostationary Navigation Overlay System (EGNOS). A high degree of correlation was found between large values of AATR and low availability of EGNOS during Solar maximum conditions. The AATR was chosen as the metric to define the ionospheric operational conditions for the EGNOS and based in this index, a set of reference test scenarios were developed to improve the EGNOS availability in front of large AATR. The result of the index adoption can be seen in Figure 1, which extends to mid 2017 the results presented in Fig. 2 of Sanz et al., 2014. The relation between the availability of EGNOS (red dotted line) and the maximum value of AATR within the corresponding day (blue line with crosses) improves after the last EGNOS update in July 2015, depicted with a yellow background. The results correspond to a fault-free receiver (as defined in ICAO, 2006) named MAS1, see Table 1.

The AATR samples ionospheric irregularities directly on the actual instantaneous GNSS signals (i.e., without any ionospheric model) similarly to Jakowski et al. (2012). The AATR has been proven as very adequate to study and characterise how the ionospheric activity degrades the accuracy (and availability) of ionospheric models, in a more correlated manner than the aforementioned planetary $D_{S T}$ or $A_{p}$ indices. Indeed, the AATR index has been adopted as a standard tool by the International Civil Aviation Organization (ICAO) for joint ionospheric studies in SBAS (ICAO, 2015).

\footnotetext{
${ }^{1}$ Project named Ionospheric Conditions and Associated Scenarios for EGNOS (ICASES), funded by the European Space Agency (ESA).
}

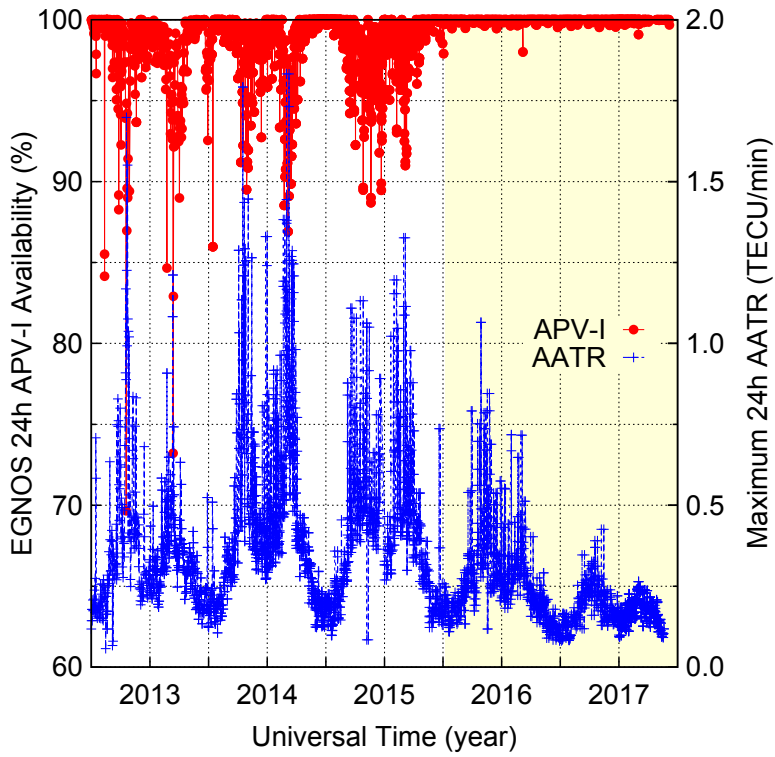

Fig. 1. Relation of the daily maximum values of AATR (blue pluses) and the daily EGNOS APV1 availability (red points) for a fault-free receiver situated in Canary Islands $(M A S 1)$. The yellow shadow indicates the EGNOS update v.2.4.1M performed between June and July of 2015 .

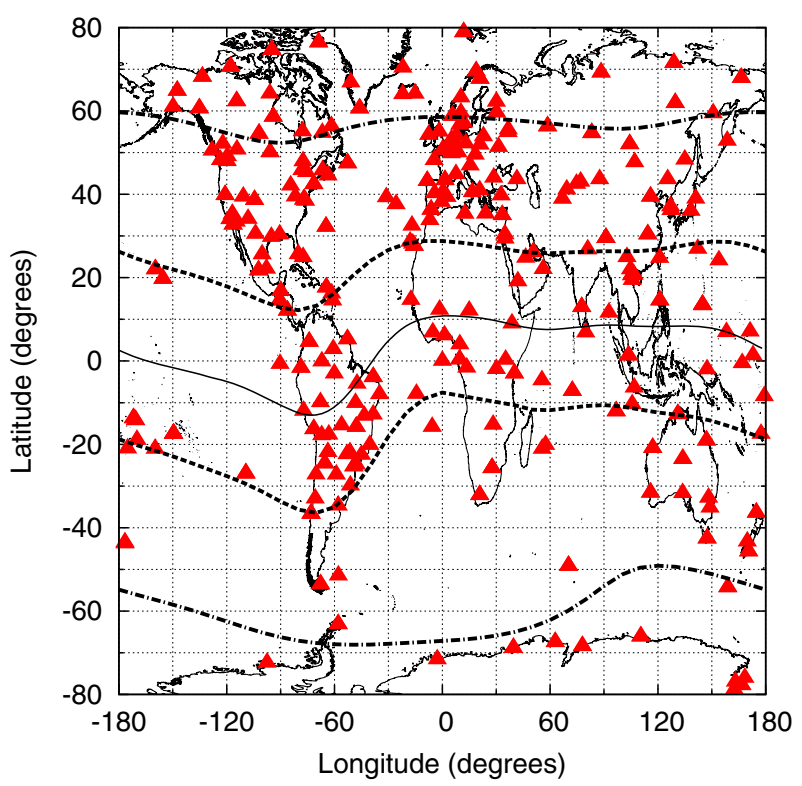

Fig. 2. Distribution of the permanent receivers (triangles) used to assess the AATR from 2002 to 2017. Black curves indicate $0^{\circ}$ (solid), $\pm 36^{\circ}$ (dashes), $\pm 60^{\circ}$ (dash-dots) MODIP latitudes.

The scientific community is also using the AATR index for ionospheric studies. It is being computed on a daily basis by different institutions for a world-wide distribution of permanent stations to monitor ionospheric disturbances (Béniguel et al., 2017). The AATR index has been used to select the periods and regions for which scintillation episodes are expected (Juan et al., 2017), prior to using other indexes (e.g. $S_{4}$ or $\sigma_{\phi}$ ) that require data at a high rate (typically at $50 \mathrm{~Hz}$ ). 
Table 1. Four character identifier and coordinates associated to a selection of the permanent stations used in the present study.

\begin{tabular}{lrrr}
\hline Station ID & Longitude $\left(^{\circ}\right)$ & Latitude $\left(^{\circ}\right)$ & MODIP $\left({ }^{\circ}\right)$ \\
\hline ACOR & -8.39 & 43.17 & 49.98 \\
DAKR & -17.49 & 14.65 & 10.16 \\
DGAR & 72.37 & -7.22 & -29.69 \\
KOUR & -52.80 & 5.21 & 16.16 \\
KRGG & 70.26 & -49.16 & -56.40 \\
MAS1 & -15.63 & 27.61 & 34.09 \\
MORP & -1.69 & 55.03 & 57.06 \\
NKLG & 9.69 & 0.40 & -24.24 \\
NOR7 & 16.25 & 58.43 & 60.02 \\
NURK & 30.08 & -1.93 & -24.45 \\
PALM & -64.05 & -64.63 & -55.11 \\
SASK & -106.40 & 52.01 & 69.59 \\
YELL & -114.48 & 62.32 & 64.19 \\
\hline
\end{tabular}

The present paper completes our previous AATR study presenting seasonal and longitudinal AATR patterns not previously discussed. Section 2 reviews the method to compute the AATR index, paying special attention to the cycle-slip detection. Section 3 describes the data set that has been used to assess the different dependencies of the AATR index. Section 4 analyses the main dependencies found in the results of AATR index. A Planetary index based on the AATR of high-latitude stations is proposed in Section 5. Section 6 shows the relation between the AATR and the SBAS performance. Finally, in Section 7, the main conclusions of this work are highlighted and summarized.

\section{The AATR index}

This section is devoted to explain how the AATR is computed. A small change of notation is introduced with respect to the original index definition in (Sanz et al., 2014), to enhance the clarity of the index derivation. Furthermore, a subsection is devoted to explain how to identify discontinuities in the carrier-phase measurements; the so-called cycle-slips. Although the cycle-slip detection do not affect to the index definition, its implementation is a relevant aspect that can affect the values of the index.

\subsection{Data preprocessing: cycle-slip detection}

A cycle-slip is a jump of an integer number of wavelengths in the ambiguity of the carrier-phase measurements between a satellite-receiver pair (Hofmann-Wellenhof et al., 2008). Under nominal conditions, these discontinuities are produced: (i) when the receiver temporary losses the lock of the GNSS signal (e.g., obstructions due to trees, buildings, bridges or mountains) and (ii) when the Signal to Noise Ratio (SNR) is low (e.g., multipath, low satellite elevation, ionospheric scintillation or high receiver dynamics).

The consecutive carrier-phase measurements between two cycle-slips belong to the same "arc", as the integer ambiguity remains constant during the arc (refer to Hofmann-Wellenhof et al., 2008, for further details). The time-derivatives involved in the AATR computation are reset every time the arc changes (i.e., after every cycle-slip). Therefore, it requires a reliable detection of such jumps: misdetections falsely increase the AATR (or ROTI) values and false-detections reduce the available data and decrease the AATR (or ROTI) values. The analysis of such tradeoff falls outside the scope of the present paper.

In order to adequately detect cycle-slips, we have used three different combinations of the carrier-phase measurements. The first detector looks for discontinuities after fitting a second degree polynomial to the geometry-free combination $(L I=L 1-L 2)$ of carrier-phase measurements. The detector threshold is larger than in the original algorithm description (Sanz et al., 2013), because we target large variations in the $L I$. The second detector uses the Melbourne Wübbena combination, as in the Turboedit algorithm (Blewitt, 1990). Finally, the third detector models the Ionosphere-Free combination of carrier-phases $\left(L_{I F}\right)$ to the centimetre level, as in the geodetic de-trending presented in (Juan et al., 2017), to detect cycleslips under ionospheric perturbed periods.

\subsection{Computation of the AATR}

Once the arcs are correctly identified, the variation of the STEC between two consecutive observations separated $\Delta t$, can be computed for a given epoch, $t$, as:

$$
\triangle \operatorname{STEC}_{i}^{j}(t)=L I_{i}^{j}(t)-L I_{i}^{j}(t-\Delta t)
$$

where $i$ denotes the receiver and $j$ the satellite. Notice that other constant or slow-varying terms that are present in the carrierphase measurements (e.g., the ambiguities and carrier hardware delays) are eliminated in the computation of (1). Conversely, misdetected cycle-slips contaminate (1) and hence the need of a reliable cycle-slip detection.

The second step is the computation of the index itself. Because we are interested in the rate of the STEC variation, we compute the instantaneous AATR:

$$
\operatorname{AATR}_{i}^{j}(t)=\frac{1}{(M(\varepsilon))^{2}} \frac{\Delta S T E C_{i}^{j}(t)}{\Delta t}
$$


where $\Delta t$ is the sampling rate of the carrier-phase measurements (typically $1 / 30 \mathrm{~Hz}$ ) and $\mathrm{M}(\varepsilon)$ is an obliquity, or slant, factor defined as the secant of the zenith angle at the mean ionospheric height, that is typically assumed at a $350 \mathrm{~km}$ of altitude. $\mathrm{M}(\varepsilon)$ projects the vertical to slant delay in a thin-shell model of the ionosphere and only depends on the satellite elevation $(\varepsilon)$ (see Sanz et al., 2013).

The purpose of dividing by the mapping function is to compare observations sampled at different elevations. The square of the mapping factor mitigates large AATR values at low elevations and, conversely, it assigns a large weight to high-elevation observations. When consecutive samples are time-differenced (e.g., ROTI or AATR) with a fixed time-step, the distance between Ionospheric Pierce Points (IPP) at lowelevation is greater than at high-elevation. Thus, even the Vertical TEC (VTEC) differences are greater at low elevation. Therefore, it makes sense to apply another correction factor (i.e., the square of the obliquity factor) to avoid this geometrical effect. In fact, when the TEC gradient (spatial variation) is considered, it is proportional to the TEC rate (time variation) if divided by the square of the mapping.

Finally, the RMS of the instantaneous $\operatorname{AATR}_{i}^{j}(t)$, is computed for a pre-defined period for all " $j$ " satellites in view from a particular station, resulting into the AATR index per a given " $i$ " receiver as:

$$
R M S_{A A T R, i}(T)=\sqrt{\frac{1}{N} \sum_{t=T}^{T+\Delta T} \sum_{j=1}^{n_{\text {sat }}(t)}\left(\operatorname{AATR}_{i}^{j}(t)\right)^{2}}
$$

where $N$ is total number of observations during the selected interval $\Delta T$ (typically one hour), after having summed all satellites in view, $n_{\text {sat }}(t)$, at every epoch $t$.

The main differences of the AATR index with respect to the ROTI are summarised as follows. The temporal resolution of the AATR is lower than of the ROTI; AATR is computed every hour, whereas the ROTI is computed every five minutes. The number of satellites involved in the AATR is greater than in the ROTI; AATR uses the rates of all the satellites in view, whereas the ROTI is computed on a satellite-per-satellite basis. In consequence, the AATR index sounds a wider area than each individualsatellitetrack, witha considerableless amountofoutput than the ROTI. This sounding can only be roughly achieved averaging values of ROTI for different receivers, for instance to generate ROTI maps in longitude/latitude grids (see Jacobsen \& Andalsvik, 2016) or magnetic local time (Cherniak et al., 2014).

\section{Experimental data}

The generation of the AATR involves dual-frequency carrier-phase measurements at low sampling rate (e.g., 1/ $30 \mathrm{~Hz}$ ). They have been downloaded in $24 \mathrm{~h}$ Receiver INdependent EXchange format (RINEX) observations files, from over one hundred permanent stations belonging to the International GNSS Service (IGS) network (see Beutler et al., 1999; Dow et al., 2009).

Figure 2 depicts the distribution of permanent stations of a typical day used for the present paper. The data collection extends from 2002 to 2017, i.e., more than one Solar Cycle, in order to assess the dependencies of the AATR with location and time at different scales (hourly, daily, and seasonal).
The third cycle-slip detector explained in Section 2.1, requires to model the carrier-phase measurement up to the centimetre level. For this purpose, it uses: (i) reference receiver coordinates, which can be obtained from the weekly Solution INdependent EXchange (SINEX) files or computed from a $24 \mathrm{~h}$ static Precise Point Positioning (PPP) processing (Kouba \& Héroux, 2001), (ii) precise satellite orbits and clocks, obtained from the IGS rapid combined product at a $900 \mathrm{~s} \mathrm{cadence}^{2}$ and (iii) the satellite and receiver antenna phase centres, obtained in the ANTenna EXchange format (ANTEX) files.

\section{Results}

This section presents the main dependencies found in the AATR, which can be divided as temporal, spatial, and related to space weather.

\subsection{Temporal dependency}

A temporal series of 17 years of AATR has been computed in order to identify the well-known dependency of ionospheric activity with the Solar activity (see for instance Liu et al., 2006). Figure 3 depicts representative examples of AATR long-term series for four IGS permanent stations globally distributed.

In the four cases, the 11 year Solar Cycle dependence can be observed as the main driver of the ionospheric activity measured through the AATR. However, the dependency has been found to be conditioned by the location of the receiver, confirming our previous research (Sanz et al., 2014). The low-latitude receivers exhibit a different periodicity (yearly in KOUR and semi-yearly in $N K L G$ ) in the maximum AATR values. This dependency is coupled with the longitude of the receiver, as it will be later analysed in Section 4.3. On the contrary, receivers located at high- or mid-latitude present large AATR values only during ionospheric storms (e.g., October 2003). In summary, the AATR long-term dependency follows in a similar manner the TEC evolution with respect to the Solar Cycle.

\subsection{Latitude dependency}

It is well-known that the ionospheric activity depends on the latitude where the receiver is located. Three different regions were identified in (Sanz et al., 2014), according to ionospheric activity. The low-, mid- and high- activity areas were determined according to the MODIP latitude, $\mu$, introduced in (Rawer, 1963):

$$
\tan \mu=\frac{\mathrm{DIP}}{\sqrt{\cos \phi}}
$$

where DIP is the inclination angle of the Earth magnetic field, $B$, and the horizontal plane at a given geographic latitude $\phi$, computed using the International Geomagnetic Reference

\footnotetext{
${ }^{2}$ Notice that the clock interpolation error is at the level of few centimetres and evolves slowly, whereas cycle-slips in the L1 or the L2 frequency produce a sudden discontinuity of 48 or $37 \mathrm{~cm}$ in the $L_{I F}$, respectively. Therefore, the satellite clock product at a cadence of 900 $\mathrm{S}$ is adequate to detect cycle-slips, as it was shown in (Juan et al., 2017).
} 


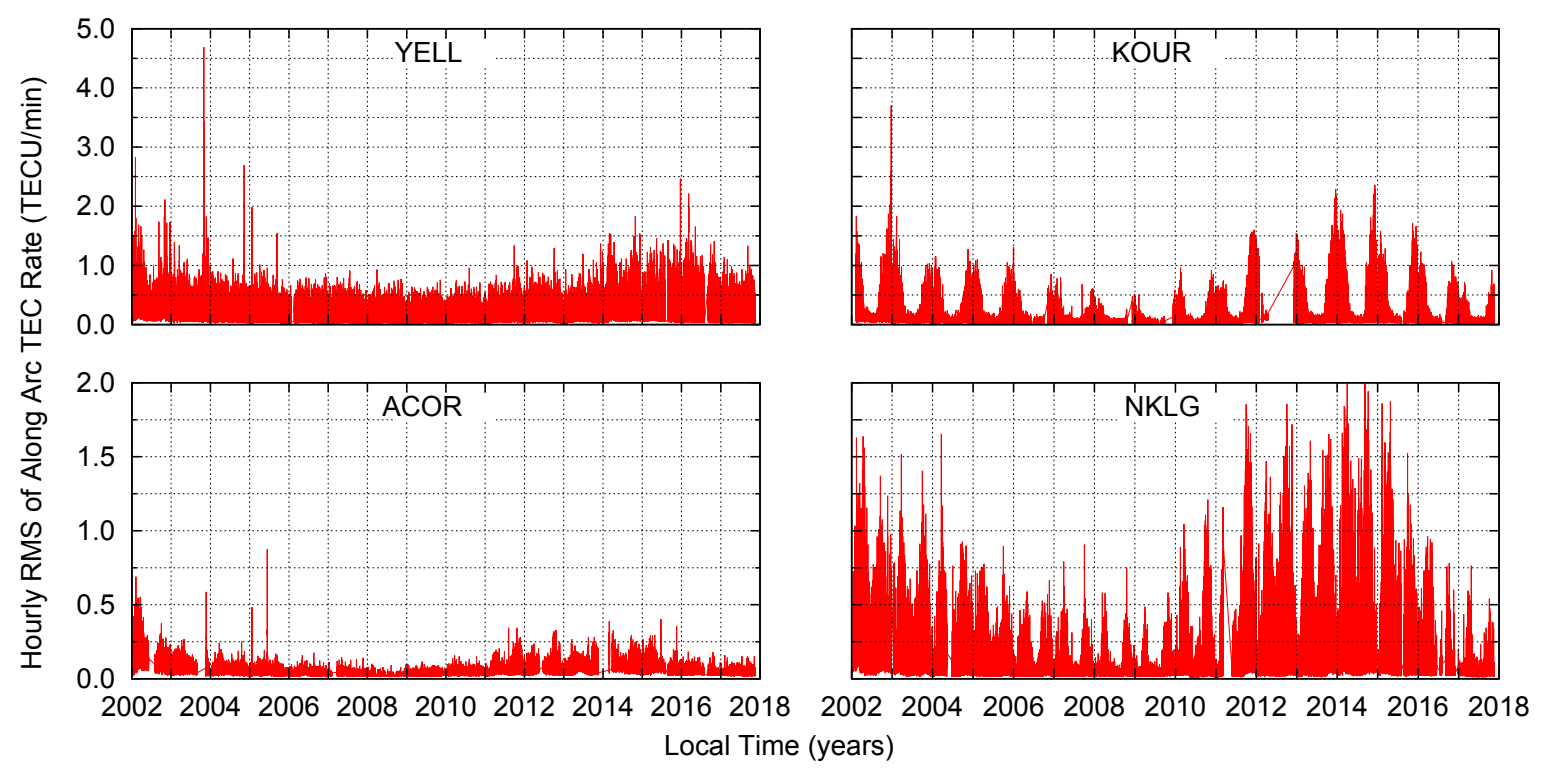

Fig. 3. From the top to bottom row and the left to right column. AATR values from 2002 to 2017 at receivers situated at high-latitude NorthAmerica (YELL), mid-latitude Europe $(A C O R)$, low-latitude South America $(K O U R)$ and low-latitude Africa $(N K L G)$. Table 1 gathers the station coordinates.

Field (IGRF) routines (Thébault et al., 2015). Figure 2 indicates with black curves different MODIP latitudes.

The classification of the AATR given in Sanz et al. (2014) is further refined in this work, after studying the time of the occurrence of large AATR values. Figure 4 depicts the percentage of AATR events greater than 0.6 TECUs/min (Sanz et al., 2014) occurred from 2002 to 2017 . Notice that the AATR occurred during post-sunset hours are distinguished (with circles) from the rest of the cases (with crosses), in order to infer the dependency of the AATR with the MODIP latitude, coupled with the Local Time (LT). The relationship is summarised as follows: at low-latitude (absolute values of MODIP lower than $36^{\circ}$ ), the greatest ionospheric activity occurs after local sunset (typically before 2 and after 18 hours LT) as it was reported in Sanz et al. (2014). On the contrary, at highlatitudes (absolute values of MODIP greater than $60^{\circ}$ ) and midlatitude (absolute values of MODIP from $36^{\circ}$ to $60^{\circ}$ ), large AATR are achieved regardless of the LT, that is during ionospheric storms or super-storms, respectively. The dependence of high-latitude AATR with space weather conditions will be later analysed in detail in Section 4.4.

\subsection{Longitude dependency}

In spite of the main dependency of the AATR is with the MODIP and with the Solar Cycle, a relation of the AATR with the longitude has also been found. For this purpose, Figure 5 depicts the seasonal variation of the AATR for the entire 2014 of five lowlatitude receivers, ordered by its longitude. It can be observed that in the westernmost receivers $K O U R$ and $D A K R$, the largest activity is concentrated from September to April, explaining the yearly periodicity previously shown in the long-term temporal dependencies of Figure 3. On the contrary, large AATR in receivers $N K L G$ and $N U R K$ occur in the vicinity of the Equinoxes (Mar 20 and Sept 23), exhibiting a semi-yearly periodicity. The relation of the AATR with the longitude is studied next.

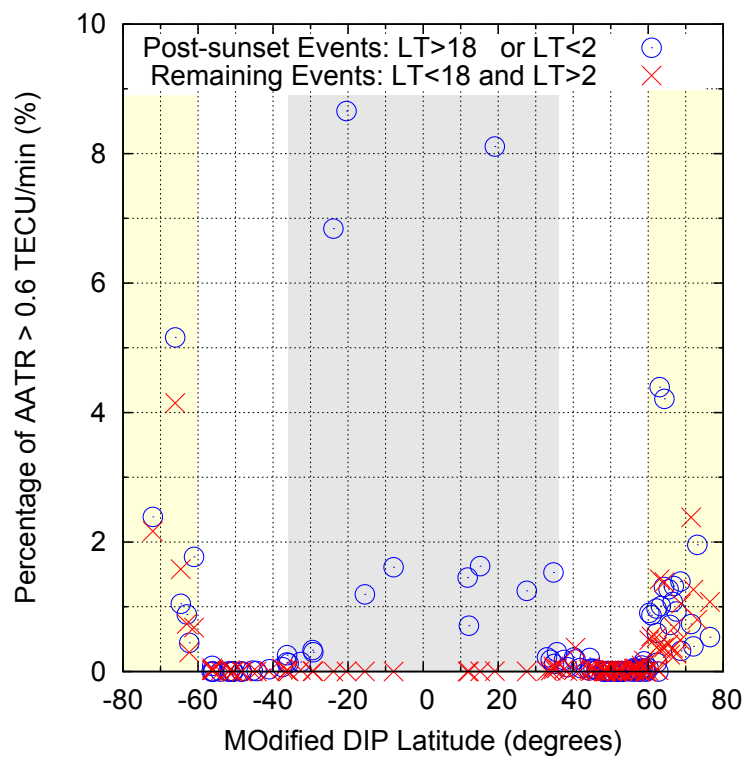

Fig. 4. AATR greater than 0.6 TECUs/min for the period from 2002 to 2017 for the receivers of Figure 2, as a function of the MOdified DIP (MODIP) latitude. The background colour indicates different ionospheric activity regions: gray (low-latitude), yellow (highlatitude), white (mid-latitude).

The seasonal-longitudinal Tsunoda, 1985 model of predicts that equatorial scintillation linked to Equatorial Plasma Bubbles (EPB), is governed by the angle $\alpha$ between the horizontal component of the geomagnetic field, $B_{H}$, and the Solar Terminator $(S T)$. Such occurrence is maximum when are $B_{H}$ and $S T$ are aligned, hence $\alpha=0^{\circ}$. The cosine of $\alpha$ is depicted in Figure 5 with a solid line, using a constant arbitrary scaling factor of 5 and a -3.5 bias. It can be observed that the alignment of $B_{H}$ and $S T$ is translated into large AATR values, 

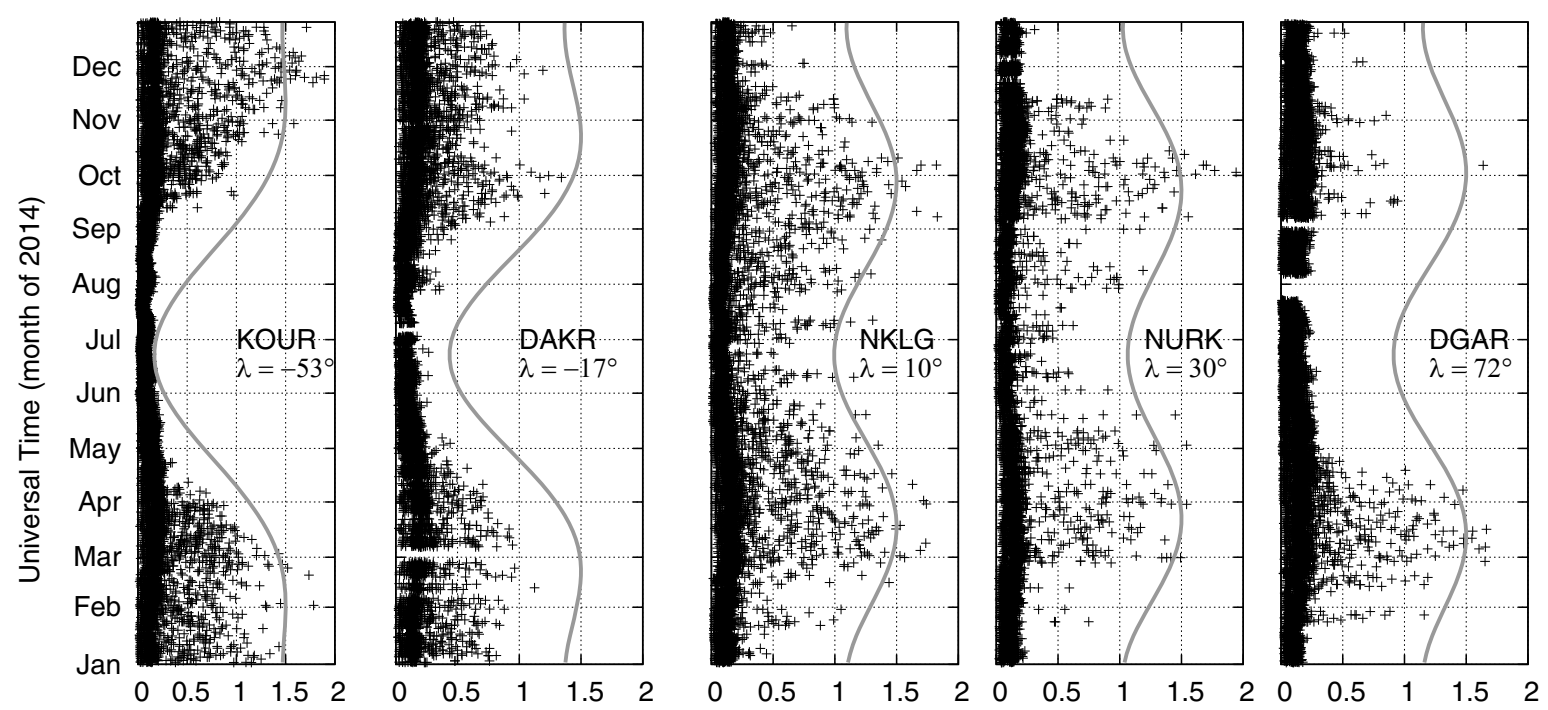

Hourly RMS of Along Arc TEC Rate (TECU/min) / 5. $\cos (\alpha)-3.5$

Fig. 5. Examples of AATR values for 2014 for the indicated low-latitude receivers (MODIP lower than $36^{\circ}$ ), as a function of the longitude. The solid grey line indicates the cosine of $\alpha$; the angle between the Earth magnetic field and the Solar Terminator.

being the maximums modulated by the cosine function. As a rule of thumb, an experimental threshold can be defined of about $\alpha=26^{\circ}$ (that is $5 \cdot \cos (\alpha)-3.5 \approx 1$ ). Indeed, values of the $\alpha$ angle smaller than $26^{\circ}$ (i.e., when the solid grey line is over 1 ) coincide with a significant enlargement of the AATR.

The characterisation of the triggers of the AATR requires a detailed study that is out of the scope of the present paper. However, the results depicted in Figure 5 suggest that large AATR values are triggered above a certain threshold of TEC. Indeed, when the TEC (or AATR) is close to such threshold, the trigger is linked to the TEC increase related to the Sun rotation. The signature of the Solar rotation period (i.e., 28 days) can be appreciated in the panels corresponding to the cases with moderate values of TEC (or AATR); specially in the $D G A R$ and NURK receivers. In the rest of the cases, the TEC (or AATR) values are far from this threshold (too high or too low, like in the KOUR receiver), and then it is more difficult to observe the relation of the AATR with the Sun rotation.

Figure 6 extends the AATR study by plotting the maximum daily AATR values on a month versus longitude map, for about 100 low-latitude receivers (i.e., $|\mathrm{MODIP}| \leq 36^{\circ}$ ). Two dark lines are superimposed on the map indicating the days when $\alpha=0^{\circ}$ at the geomagnetic equator. It can be observed that large AATR occur nearly the $\alpha=0^{\circ}$ lines. Furthermore, the temporal separation of the $\alpha=0^{\circ}$ events is found to be minimum in the Pacific sector (about 125 days), and maximum in the South Atlantic Anomaly (SAA) (about 296 days), in agreement to the five examples presented in Figure 3. This AATR longitudinal dependency coupled with the temporal dependency of $\alpha=0^{\circ}$, agrees with the occurrence map of EPB derived from lowaltitude polar-orbiting satellites (Burke et al., 2004).

\subsection{Space weather}

This section presents the interrelation of the AATR with different space weather conditions. We have selected the geomagnetic storm of March 17 of 2015, i.e., St. Patrick's day,

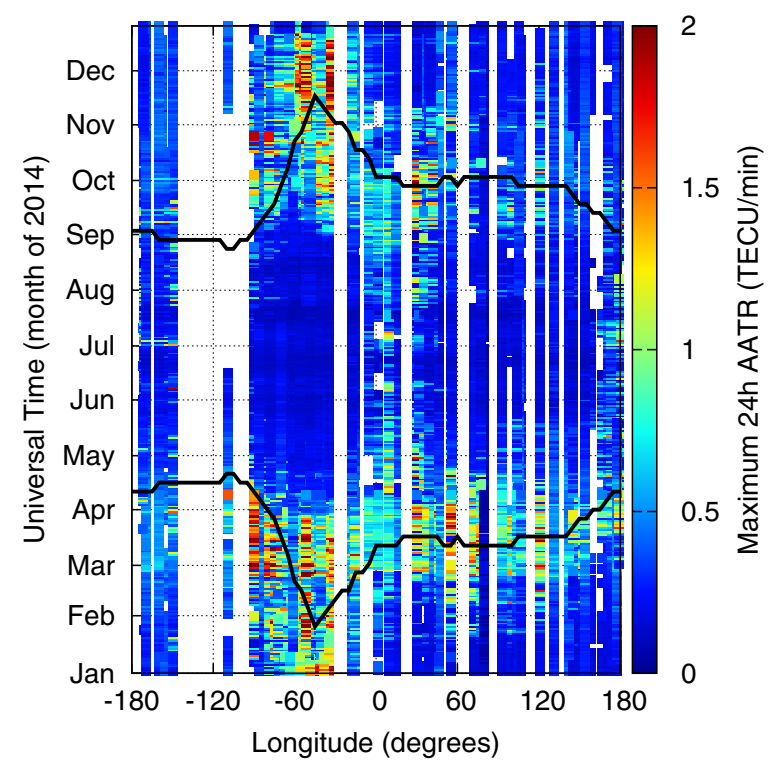

Fig. 6. Maximum daily AATR values for 2014 as a function of the longitude and month, for receivers situated at low-latitude ( $\mid$ MODIP $\mid \leq 36^{\circ}$ ). The solid black lines indicates the two times per year when the horizontal component of the Earth magnetic field is aligned the Solar Terminator (thence $\alpha=0^{\circ}$ ), at the geomagnetic equator.

which has been extensively treated in the literature (see for instance, Jacobsen \& Andalsvik, 2016; Béniguel et al., 2017, and references therein). For this purpose, Figure 7 is divided in panels showing different phenomena related to the Sun (panel a), to the interplanetary medium (panel b), to the ionosphere (panel c), and to the Earth (panel d).

The origin of the geomagnetic storm was a Coronal Mass Ejection (CME) occurred from 2:00 to 2:30 on March 15, according to the NOAA Space Weather Prediction Centre 

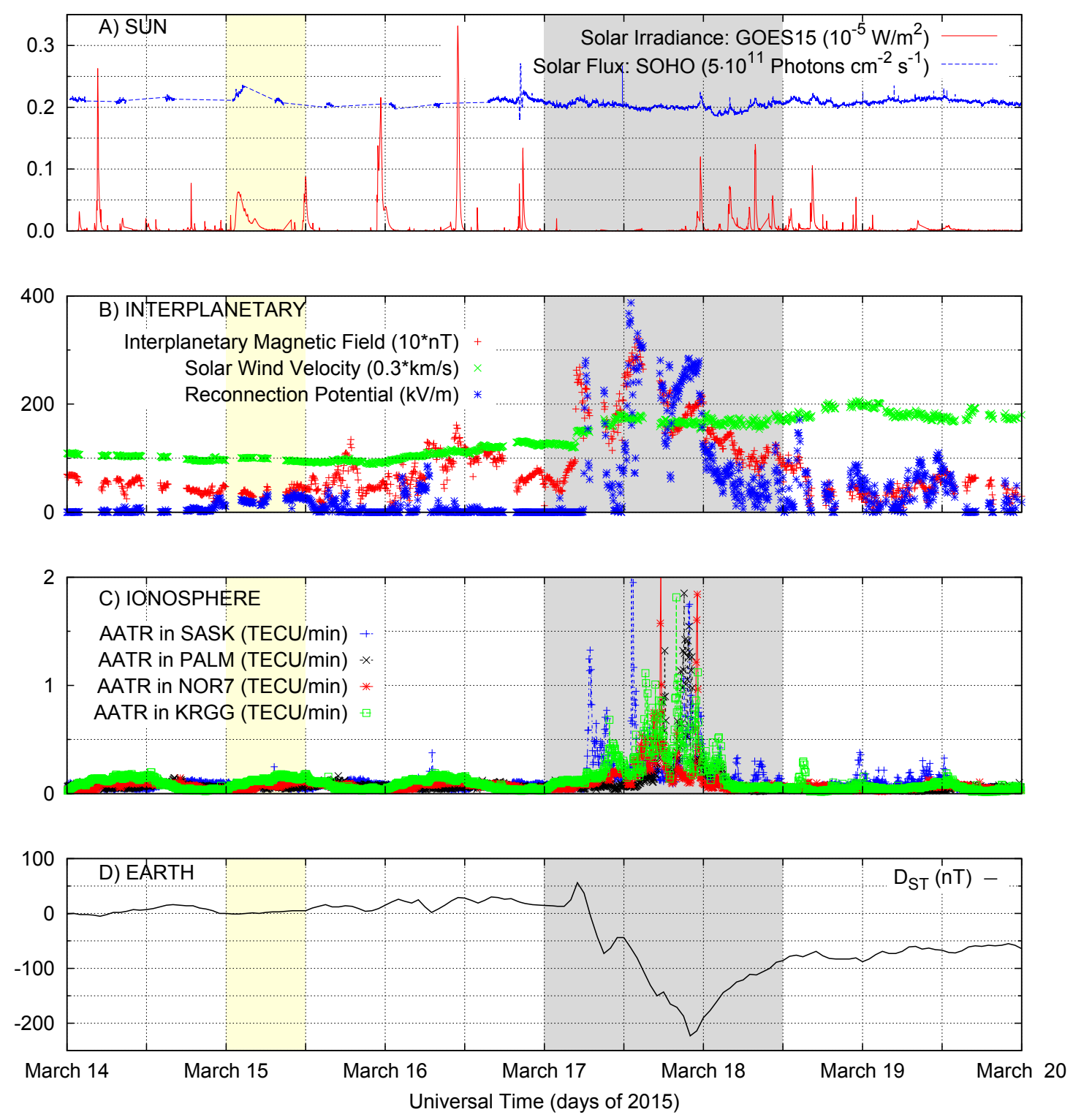

Fig. 7. AATR relation with different space weather conditions. The top plot depicts the solar flares sampled by different sun-observing satellites. The second panel depicts the variation of the IMF and Solar wind at the magnetosphere, together with the reconnection potential. The third panel depicts some AATR at different receivers distributed in both hemispheres, see Table 1 . The bottom panel, depicts the geomagnetic index $D_{S T}$.

(http://www.swpc.noaa.gov/). This event corresponds to the yellow shadow in the panel (a) of Figure 7, where a peak can be observed in the Solar irradiance (red solid line) measured by the Geostationary Operational Environmental Satellite (GOES) (Hill et al., 2005) and in the Solar flux (blue dashed line) sampled by the SOlar Helioscopic Observatory (SOHO) (Judge et al., 1998).

The panel (b) of Figure 7 depicts the coupling between Interplanetary Magnetic Field (IMF) and the magnetosphere, that is the space environment located from two to several Earth radii. The IMF lines interconnect with terrestrial magnetic field lines, accumulating magnetic flux into the magnetosphere and expanding the polar caps (Milan et al., 2012). This effect is proportional to (i) the speed of the Solar wind (depicted with green crosses), (ii) to the intensity of the transverse component of the IMF (depicted with red pluses) and (iii) to the IMF orientation. These three datum have been downloaded from the OMNIWeb website (King \& Papitashvili, 2005), to compute the day-side reconnection voltage (blue solid line) using the expression by Milan et al. (2012). When the reconnection potential is large enough, energetic particles precipitate from the magnetosphere towards the ionosphere.

The panel (c) of Figure 7 depicts the effect of the CME arrival to the Earth ionosphere two days later. In order to track the storm with a greater temporal resolution, the RMS of the AATR has been computed every five minutes, instead of every hour. It can be observed that the ionospheric perturbation is sampled by the AATR of several mid-latitude receivers situated at both hemispheres, see Table 1. As commented in Section 4.2, mid-latitude receivers present large AATR values only during large storms. Indeed, St. Patrick storm was "severe" according to the NOAA space weather Scales (Poppe, 2000). 

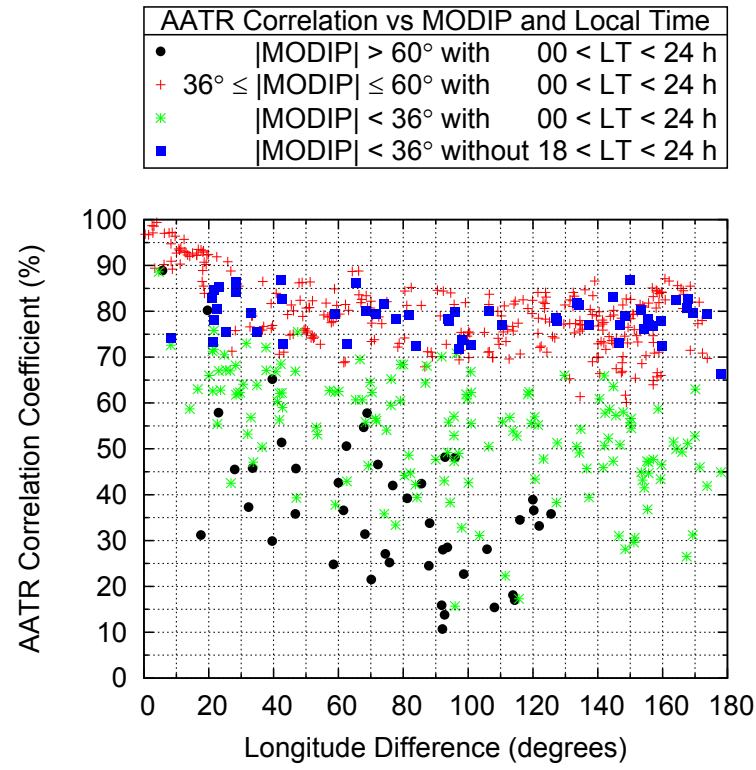

Fig. 8. Correlation of AATR with longitude for different MODIP bands and $24 \mathrm{~h}$ of LT. For the equatorial band, the correlation index is also presented excluding the post-sunset LTs.

Finally, the panel (d) of Figure 7 shows the perturbation of the geomagnetic index $D_{S T}$, computed from Earth observations on a hourly basis. It can be seen the the main phase of the storm (negative $D_{S T}$ variation from +56 to $-223 n T$ ) coincides with the period of large AATR values. However, once the reconnection voltage (panel $b$ ) decreases during the first hours of March 18, the AATR activity vanishes. On the contrary, the $D_{S T}$ remains perturbed for some days after the storm.

\section{5 planetary index based on the AATR}

This section investigates the feasibility of defining a planetary storm ionospheric index based on the AATR and its capability to sample several phenomena like space weather events.

\subsection{Correlation of AATR}

In order to define such a planetary index, the first magnitude to quantify is the degree of correlation of the AATR values of receivers situated at different longitudes. In order to accommodate the diurnal variation of the ionosphere, the AATR are compared at the same LT. Indeed, receivers located in similar MODIPs sharing a constant LT can be assumed to have similar physical conditions (Rawer, 1963).

Figure 8 depicts the correlation coefficient for the AATR under these assumptions. Different behaviours are clearly appreciated in the three regions identified in Section 4.2. The high-latitude region, depicted with black dots, presents the worse correlation results, rapidly decaying to a small correlation ratio with increasing longitude difference. The mid-latitude region, depicted with red pluses, presents the largest correlation ratio, maintained at around $80 \%$ for all longitude differences (i.e., at a planetary scale). The lowlatitude region, depicted with green asterisks, presents a mid-

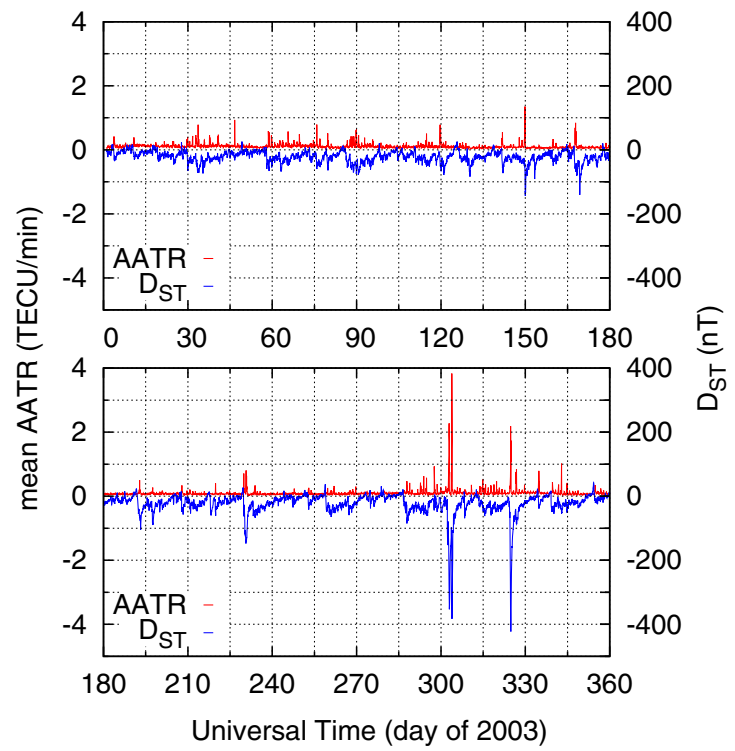

Fig. 9. Relation of the mean AATR computed from receivers situated around $|\mathrm{MODIP}| \approx 50^{\circ}$ and LTs around the noon, with the $D_{S T}$. The first half of the year 2003 is depicted in the top panel, while the second half in the bottom panel.

correlation when all LTs are considered altogether. Nevertheless, if LTs after the local sunset are excluded (blue squares), the correlation increases to the level of the mid-latitude region.

\subsection{Index proposal}

The correlations observed for the AATR can be exploited to define a planetary index. However, the selection of the receivers involved in the computation of the planetary storm index needs to be carefully done. Equatorial and polar receivers are not suitable as they exhibit a correlation too low (see Fig. 8). On the contrary, most of the mid-latitude receivers are located in a too-quiet ionospheric region (see Fig. 3). Therefore, receivers used for the storm index are selected within the upper range of the middle latitude. Indeed, the AATR of receivers located around $|\mathrm{MODIP}| \approx 50^{\circ}$ are specially sensitive to the ionospheric perturbations related with space weather, in a similar manner than the planetary index $D_{S T}$. Therefore, the index is proposed as the average of AATR from receivers around the noon (e.g., 12-14 LT) and located at latitudes in the sub-aurora region $\left(45^{\circ} \leq|\mathrm{MODIP}| \leq 55^{\circ}\right)$ :

$$
\overline{A A T R}=\frac{1}{N_{A A T R}} \sum_{L T=12}^{14} \sum_{M O D=45}^{55} A A T R_{M O D}(L T)
$$

where $N_{\text {AATR }}$ is total number of AATR considered for the period. Notice that the computation of the average AATR index defined in (5) requires an enough number of receivers placed at the sub-auroral region. However, the current distribution of the IGS network provides access to hundreds of permanent stations at such latitudes, including tens of them in real-time.

Figure 9 depicts the results of the AATR (red line) for the entire 2003, together with the $D_{S T}$ index (blue line). It can be observed a coincidence on time between the peaks of both 

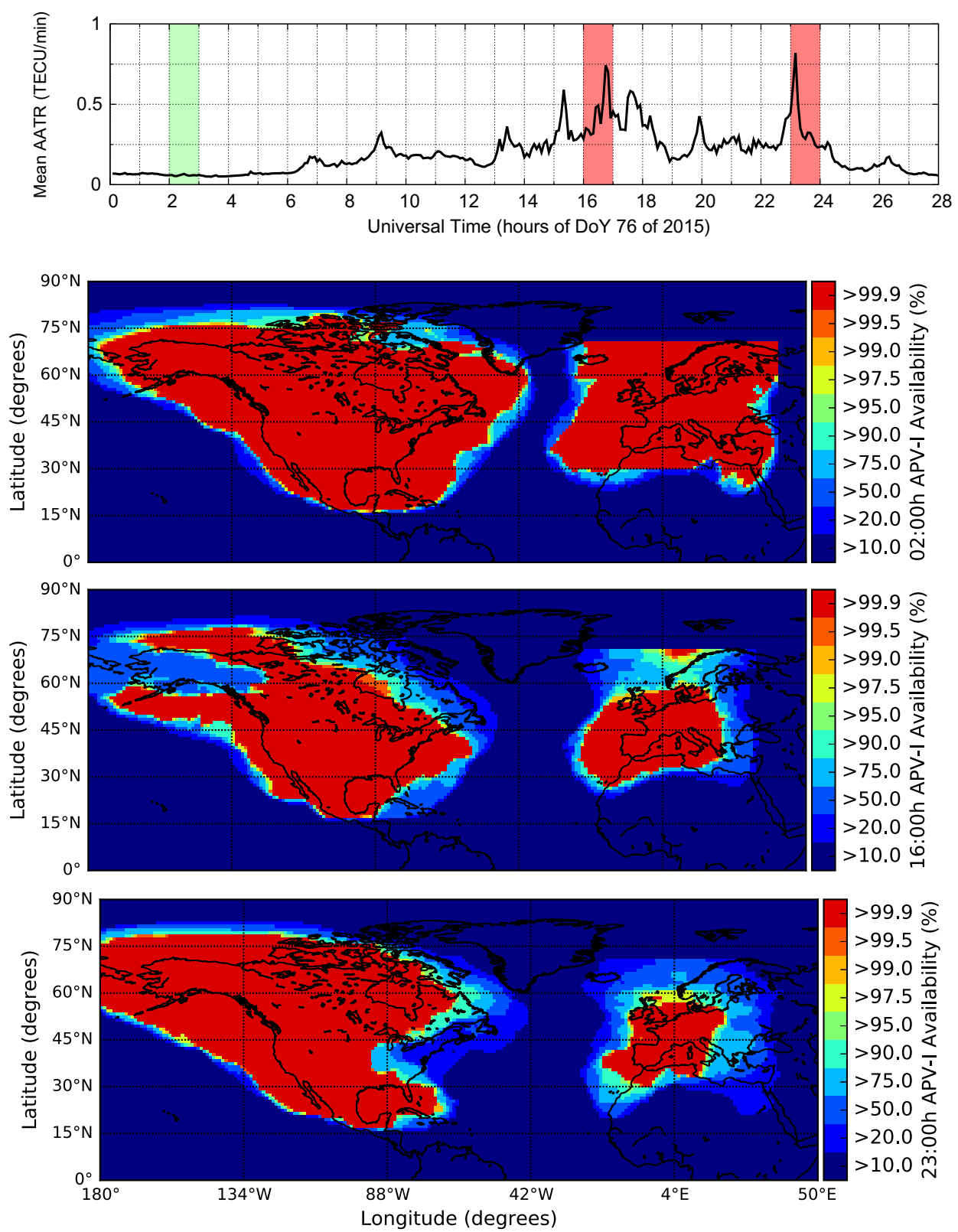

Fig. 10. Relation between the mean value of AATR of sub-auroral receivers (top panel) and the SBAS availability during a nominal hour ( $2 \mathrm{~h}$, second panel) and during two storm hours (16h, third and $23 \mathrm{~h}$, bottom panel) within DoY 76 of 2015 (March 17). The colour bar depicts the percentage of epochs where the Protection Levels are under the Alarm Limits of $40 \mathrm{~m}$ (horizontal) and $50 \mathrm{~m}$ (vertical) simultaneously. Each pixel represents a square of 1 degree of resolution, without applying any interpolation.

indexes. However, it should be noticed that the proposed AATR index presents two differences in front of the $D_{S T}$ index: (i) the AATR index is not affected by recovering periods of geomagnetic storms, and (ii) there is not need of calculating a yearly or daily means for discounting long-term signatures, as it is done for computing the $D_{S T}$.

\section{Application of the AATR to navigation}

This section presents the application of the proposed storm index to the European and American SBAS for the aforementioned St. Patrick storm. We have used our open-source gLAB
(Sanz et al., 2012) to compute the hourly availability maps for the Wide Area Augmentation System (WAAS) (over North America) and EGNOS (over Europe) depicted in Figure 10. The availability is computed as the percentage of epochs within the hour in which the Protection Levels are under the Alarm Limits of $40 \mathrm{~m}$ (horizontal) and $50 \mathrm{~m}$ (vertical) simultaneously (see RTCA, 2006, for additional details). The maps were computed in a single process run (i.e., without merging individual maps from EGNOS and WAAS), thanks to the update performed in ESA (2016). The SBAS corrections correspond to the ones broadcast by the geostationary satellites 133 (WAAS) and 120 (EGNOS), that were kindly provided by the Centre National d'Etudes Spatiales (CNES) on our request. 
The top panel of Figure 10 depicts the mean global AATR index with a solid black line. It can be observed that the perturbation in the index starts at $6 \mathrm{~h}$, reaches two pronounced peaks at $16 \mathrm{~h}$ and at $23 \mathrm{~h}$, and after $24 \mathrm{~h}$, the values returns to a nominal (unperturbed) value. Then, we have selected the $2 \mathrm{~h}$ (green background) as representative of the nominal performance of the SBAS, whereas the hours associated to the two peaks (red backgrounds) are used to assess both SBAS during severe storm conditions.

Indeed, the second panel of Figure 10 depicts availabilities close to $100 \%$ (red colours) on the coverage area of each SBAS. On the contrary, the third and fourth panels depict a severe degradation of the availability (blue colours) on the coverage regions of both SBAS at different hours. WAAS and EGNOS return to a nominal performances after $24 \mathrm{~h}$ (not depicted), in agreement with small AATR values. On the contrary, the $D_{S T}$ index is kept perturbed (see bottom panel of Fig. 7).

The depicted example illustrates how the AATR index (or its average AATR) helps to distinguish problems related with the ionospheric activity from other error sources (e.g., satellite clocks) which can affect to the EGNOS service. This is the reason why EGNOS is using the AATR routinely as a metric for characterizing the ionospheric conditions.

\section{Conclusions}

There are four important conclusions from the research presented in this paper.

- The AATR is a reliable index for ionospheric studies, that can be computed from actual GNSS carrier-phase measurements at a low sampling rate.

- The AATR follows the 11 year period of the Solar Cycle. A seasonal dependency has been found, being modulated by the angle between the horizontal component of the Earth magnetic field and the Solar Terminator.

- The AATR are largest at low-latitudes but are circumscribed to post-sunset time. Excluding such period, the equatorial ionospheric activity is moderate and as spatially (i.e., longitudinally) correlated as in mid-latitude.

- A planetary storm index $\overline{\text { AATR }}$ can be defined using the mean value of AATR of sub-auroral receivers, which are correlated with space weather events. The index can be used to see degradations of current SBAS.

Acknowledgements. This work has been supported by the Spanish Ministry of Science and Innovation project CGL2015-66410-P, the European Space Agency projects ICASES and $g$ EGNOS, and the European Union's Horizon 2020 research and innovation programme under grant agreement No 776011 (TechTIDE project). The authors acknowledge the use of data from the IGS, from the CNES, and from the NASA Goddard Space Flight Center. The editor thanks the referees Mamoru Ishii and Simon Banville for their assistance in evaluating this paper.

\section{References}

Béniguel Y, Cherniak I, Garcia-Rigo A, Hamel P, Hernández-Pajares M, et al. 2017. MONITOR ionospheric network: two case studies on scintillation and electron content variability. Ann Geophys 35: 377-391. DOI: 10.5194/angeo-35-377-2017.

Beutler G, Rothacher M, Schaer S, Springer T, Kouba J, Neilan R. 1999. The International GPS Service (IGS): an interdisciplinary service in support of Earth sciences. Adv Space Res 23: 631-653, DOI:10.1016/S0273-1177(99)00160-X.

Blewitt G. 1990. An automatic editing algorithm for GPS data. Geophys Res Lett 17: 199-202, DOI:10.1029/GL017i003p00199.

Briggs B, Parkin I. 1963. On the variation of radio star and satellite scintillations with zenith angle. J Atmos Terr Phys 25: 339-366, DOI:10.1016/0021-9169(63)90150-8.

Burke WJ, Gentile LC, Huang CY, Valladares CE, Su SY. 2004. Longitudinal variability of equatorial plasma bubbles observed by DMSP and ROCSAT-1. J Geophys Res: Space Phys 109: 1-12, DOI:10.1029/2004JA010583.

Cherniak I, Krankowski A, Zakharenkova I. 2014. Observation of the ionospheric irregularities over the northern hemisphere: methodology and service. Radio Sci 49: 653-662, DOI:10.1002/ 2014RS005433.

Datta-Barua S, Walter T, Altshuler E, Blanch J, Enge P. 2005. Dst as an indicator of potential threats to WAAS integrity and availability. In: Proceedings of ION GPS 2005. California, USA, pp. 2365 2373. http://www.ion.org/publications/abstract.cfm?articleID= 6442.

Doherty P, Raffi E, Klobuchar J, El-Arini M. 1994. Statistics of time rate of change of ionospheric range delay. In: Proceedings of the 7th International Technical Meeting of the Satellite Division of The Institute of Navigation (ION GPS 1994). Salt Lake City, UT USA, pp. 1589-1598. https://www.ion.org/publications/abstract.cfm?arti cleID $=3981$.

Dow J, Neilan RE, Rizos C. 2009. The international GNSS service in a changing landscape of global navigation satellite systems. J Geod 83: 191-198, DOI:10.1007/s00190-008-0300-3.

ESA. 2016. gEGNOS: gLAB upgrade for EGNOS data processing, http://www.gage.upc.edu/forum/glab-upgrade-egnos-data-process ing-gegnos.

Gulyaeva TL, Stanislawska I. 2008. Derivation of a planetary ionospheric storm index. Ann Geophys 26: 2645-2648, DOI:10.5194/angeo-26-2645-2008.

Hathaway DH. 2010. The Solar Cycle. Living Rev Sol Phys 7: 1, DOI:10.12942/lrsp-2010-1.

Hill SM, Pizzo VJ, Balch CC, Biesecker DA, Bornmann P, et al. 2005. The NOAA Goes-12 Solar X-Ray Imager (SXI) 1. Instrument, operations, and data. Sol Phys 226: 255-281, DOI:10.1007/ s11207-005-7416-x.

Hofmann-Wellenhof B, Lichtenegger H, Wasle E. 2008. GNSS. Global Navigation Satellite Systems, Springer, Vienna, Austria, ISBN 978-3-211-73012-6, DOI: 10.1007/978-3-211-73017-1.

ICAO. 2006. Annex 10 to the Convention on International Civil Aviation: Aeronautical Telecommunications, Montreal, Canada, ISBN 92-9194-772-5, https://store.icao.int/annex-10-aeronauticaltelecommunications-volume-ii-english-printed.html. 
ICAO. 2015. Report of the fifth meeting of Ionospheric Studies Task Force (ISTF/5), Okinawa, Japan, http://www.icao.int/APAC/Meet ings/2015\%20ISTF5/ISTF5\%20Report\%20-\%20final.pdf.

IEEE. 1997. Institute of Electrical and Electronics Engineers Standard 211, New York, USA, ISBN 0-7381-0223-7, https://standards.ieee. org/findstds/standard/211-1997.html.

Jacobsen K, Andalsvik Y. 2016. Overview of the 2015 St. Patricks day storm and its consequences for RTK and PPP positioning in Norway. J Space Weather Space Clim 6: A9, DOI:10.1051/swsc/2016004.

Jakowski N, Borries C, Wilken V. 2012. Introducing a disturbance ionosphere index. Radio Sci 47: 1-9, DOI: 10.1029/ 2011RS004939.

Jakowski N, Stankov S, Schlueter S, Klaehn D. 2006. On developing a new ionospheric perturbation index for space weather operations. $A d v$ Space Res 38: 2596-2600, Middle and upper atmospheres, active experiments, and dusty plasmas, DOI:10.1016/j.asr.2005.07.043.

Juan J, Aragon-Angel A, Sanz J, González-Casado G, Rovira-Garcia A. 2017. A method for scintillation characterization using geodetic receivers operating at $1 \mathrm{~Hz}$. J Geod 91: 1383-1397, DOI:10.1007/ s00190-017-1031-0.

Judge DL, McMullin DR, Ogawa HS, Hovestadt D, Klecker B, et al. 1998. First solar EUV irradiances obtained from SOHO by the CELIAS/SEM, Springer, Dordrecht, Netherlands, pp. 161-173, ISBN 978-94-011-5000-2, DOI: 10.1007/978-94-011-5000-2_12.

King JH, Papitashvili NE. 2005. Solar wind spatial scales in and comparisons of hourly wind and ACE plasma and magnetic field data. J Geophys Res: Space Phys 110: 1-8 DOI:10.1029/ 2004JA010649.

Kouba J, Héroux P. 2001. Precise point positioning using IGS orbit and clock products. GPS Solut 5: 12-28, DOI: 10.1007/ PL00012883.

Liu L, Wan W, Ning B, Pirog OM, Kurkin VI. 2006. Solar activity variations of the ionospheric peak electron density. J Geophys Res: Space Phys 111: 1-13, DOI:10.1029/2006JA011598.

Milan SE, Gosling JS, Hubert B. 2012. Relationship between interplanetary parameters and the magnetopause reconnection rate quantified from observations of the expanding polar cap. J Geophys Res: Space Phys 117: 1-16, DOI:10.1029/2011JA017082.

Pi X, Mannucci AJ, Lindqwister UJ, Ho CM. 1997. Monitoring of global ionospheric irregularities using the worldwide GPS Network. Geophys Res Lett 24: 2283-2286, DOI:10.1029/97GL02273.

Poppe BB. 2000. New scales help public, technicians understand space weather. Eos, Trans Am Geophys Union 81: 322-328, DOI: 10.1029/00EO00247.
Pullen S, Park YS, Enge P. 2009. Impact and mitigation of ionospheric anomalies on ground-based augmentation of GNSS. Radio Sci 44: 1-10, DOI:10.1029/2008RS004084.

Rawer K. 1963. Propagation of decameter waves (HF-band) in meteorological and astronomical influences on radio wave propagation. Landmark B (Ed.), Pergamon Press, New York.

Rostoker G. 1972. Geomagnetic indices. Rev Geophys 10: 935-950, DOI: 10.1029/RG010i004p00935.

RTCA. 2006. Minimum operational performance standards for global positioning system/wide area augmentation system airborne equipment. RTCA document 229-D.

Sanz J, Rovira-Garcia A, Hernández-Pajares M, Juan M, VenturaTraveset J, López-Echazarreta C, Hein G. 2012. The ESA/UPC GNSS-Lab Tool (gLAB): an advanced educational and professional package for GNSS data processing and analysis. In: Proceedings of Toulouse Space Show 2012, 4th International Conference on Space Applications. Toulouse, France, http://gage6. upc.es/gAGE_WEB/papers/2012/FP_SA-133.pdf.

Sanz J, Juan J, Hernández-Pajares M. 2013 . GNSS data processing, Vol. I: fundamentals and algorithms. ESA communications, ESTEC TM-23/1, Noordwijk, Netherlands, ISBN 978-92-9221-886-7, http://www.navipedia.net/GNSS_Book/ESA_GNSSBook_TM-23_Vol_I.pdf.

Sanz J, Juan J, González-Casado G, Prieto-Cerdeira R, Schlueter S, Orús R. 2014. Novel ionospheric activity indicator specifically tailored for GNSS users. In: Proceedings of ION GNSS+ 2014. Tampa, Florida (USA), pp. 1173-1182. http://www.ion.org/ publications/abstract.cfm?.jp=p\&articleID=12269.

Thébault E, Finlay CC, Beggan CD, Alken P, Aubert J, et al. 2015. International Geomagnetic Reference Field: the 12th generation. Earth, Planets Space 67: 79, DOI:10.1186/s40623-015-0228-9.

Tsunoda RT. 1985. Control of the seasonal and longitudinal occurrence of equatorial scintillations by the longitudinal gradient in integrated E region Pedersen conductivity. J Geophys Res: Space Phys 90: 447-456, DOI:10.1029/JA090iA01p00447.

Walter T, Hansen A, Blanch J, Enge P, Mannucci T, et al. 2001. Robust detection of ionospheric irregularities. Navigation 48: 89-100, DOI:10.1002/j.2161-4296.2001.tb00231.x.

Yeh C, Chao-Han L. 1982. Radio wave scintillations in the ionosphere. Proc IEEE 70: 324-360, DOI:10.1109/ PROC.1982.12313.

Zolesi B, Cander LR. 2014. Ionospheric prediction and forecasting, Springer Geophysics, Heidelberg, Germany, ISBN 978-3-642-38429-5, DOI:10.1007/978-3-642-38430-1.

Cite this article as: Juan JM, Sanz J, Rovira-Garcia A, González-Casado G, Ibáñez D, Perez RO. 2018. AATR an ionospheric activity indicator specifically based on GNSS measurements. J. Space Weather Space Clim. 8: A14 